\title{
Improved SAR Amplitude Image Offset Measurements for Deriving Three-Dimensional Coseismic Displacements
}

\author{
Teng Wang and Sigurjón Jónsson
}

\begin{abstract}
Offsets of synthetic aperture radar (SAR) images have played an important role in deriving complete threedimensional (3-D) surface displacement fields in geoscientific applications. However, offset maps often suffer from multiple outliers and patch-like artifacts, because the standard offsetmeasurement method is a regular moving-window operation that does not consider the scattering characteristics of the ground. Here, we show that by focusing the offset measurements on predetected strong reflectors, the reliability and accuracy of SAR offsets can be significantly improved. Application to the 2011 Van (Turkey) earthquake reveals a clear deformation signal from an otherwise decorrelated interferogram, making derivation of the 3-D coseismic displacement field possible. Our proposed method can improve mapping of coseismic deformation and other ground displacements, such as glacier flow and landslide movement when strong reflectors exist.
\end{abstract}

Index Terms-Pixel offsets, synthetic aperture radar (SAR), three-dimensional (3-D) displacement.

\section{INTRODUCTION}

W HILE interferometric synthetic aperture radar (InSAR) has become the main technique to study earthquake deformation, it provides only one-dimensional (1-D) measurements along the satellite line-of-sight (LOS) look direction and often suffers from interferometric decorrelation due to changes in surface reflection characteristics (e.g., caused by vegetation growth) or large displacement gradients. To reduce decorrelation in coseismic interferograms, researchers select pre- and postearthquake images with as small temporal span and perpendicular baselines as possible, filter the interferograms, and then simply mask out the decorrelated areas before using the data in modeling, e.g., in [1], which often leaves only partial coverage of the coseismic deformation field. The problem of using only 1-D LOS displacements in fault source modeling is that 1-D displacement data have limited model resolution, resulting in strong tradeoffs between model parameters, e.g., between dip and rake of the fault [2]. These tradeoffs can be significantly reduced when using InSAR data from both ascending

Manuscript received September 15, 2014; revised November 25, 2014; accepted December 17, 2014.

The authors are with King Abdullah University of Science and Technology, Thuwal 23955-6900, Saudi Arabia (e-mail: teng.wang@kaust.edu.sa).

Color versions of one or more of the figures in this paper are available online at http://ieeexplore.ieee.org.

This paper has supplementary downloadable multimedia material available at http://ieeexplore.ieee.org provided by the authors. This includes a PDF that lists the point-target offset results and the 3-D coseismic displacements (Figs. $\mathrm{S} 1-\mathrm{S} 5)$. This material is $12,892 \mathrm{~KB}$ in size.

Digital Object Identifier 10.1109/JSTARS.2014.2387865 and descending orbits, although two independent LOS observations cannot resolve the full three-dimensional (3-D) surface displacement field.

Pixel offset measurements between two SAR amplitude images can provide unambiguous ground displacement measurements in both the LOS direction and parallel to the alongtrack (azimuth) satellite flying direction [3]. The accuracy of pixel-offset measurements depends on the cross-correlation quality, and can be in the order of one-tenth of the pixel spacing [4], [5]. Although the offset measurements are less accurate than the InSAR phase measurements, they allow for the full 3-D coseismic displacement field to be derived, when datasets from both ascending and descending orbits are combined [6]-[9]. In earlier studies, only the azimuth pixel offsets were considered in combination with the InSAR phase measurements to derive 3-D displacements, e.g., in [6] and [7]. The range offsets, on the other hand, have usually been discarded, as they quantify displacement in the same LOS direction as the InSAR phase measurement but with much lower accuracy. Range offsets have, however, proven to be useful when the ground displacements are large and for quantifying displacement across major discontinuities, e.g., in [8], [10], and [11]. In addition, range offsets have been used to improve InSAR observations [12] and to augment InSAR measurements to obtain near-field coseismic displacements [13].

Since the COSMO-SkyMed (CSK) and TerraSAR-X (TSX) X-band (wavelength $\lambda=3.1 \mathrm{~cm}$ ) satellites were launched in 2007-2010, increasing amounts of high-resolution SAR data are being acquired. The pixel spacing of these high-resolution SAR images is around $1 \mathrm{~m}$, which means that the accuracy of both the range and azimuth offset measurements is significantly better than that of SAR images from the C-band $(\lambda=5.6 \mathrm{~cm})$ ERS and Envisat satellites and the L-band $(\lambda=23.6 \mathrm{~cm})$ ALOS satellite, whose pixel spacing is $4-8 \mathrm{~m}$. It has been argued that, under optimal conditions, the accuracy of TSX range offsets can even be comparable to L- and C-band InSAR phase measurements [4]. In addition, the accurate orbital information of the TSX satellite allows for centimeter-level absolute range measurements on corner reflectors and other ideal targets, after carefully removing atmospheric and solid tides effects [14]. The X-band InSAR data, on the other hand, decorrelate in a relatively short time due to their short wavelength, often leading to incoherent coseismic interferograms and/or to aliased fringes, limiting the usability of these data. As a result, offsets of X-band SAR images are increasingly being used to study ground displacements due to a variety of 


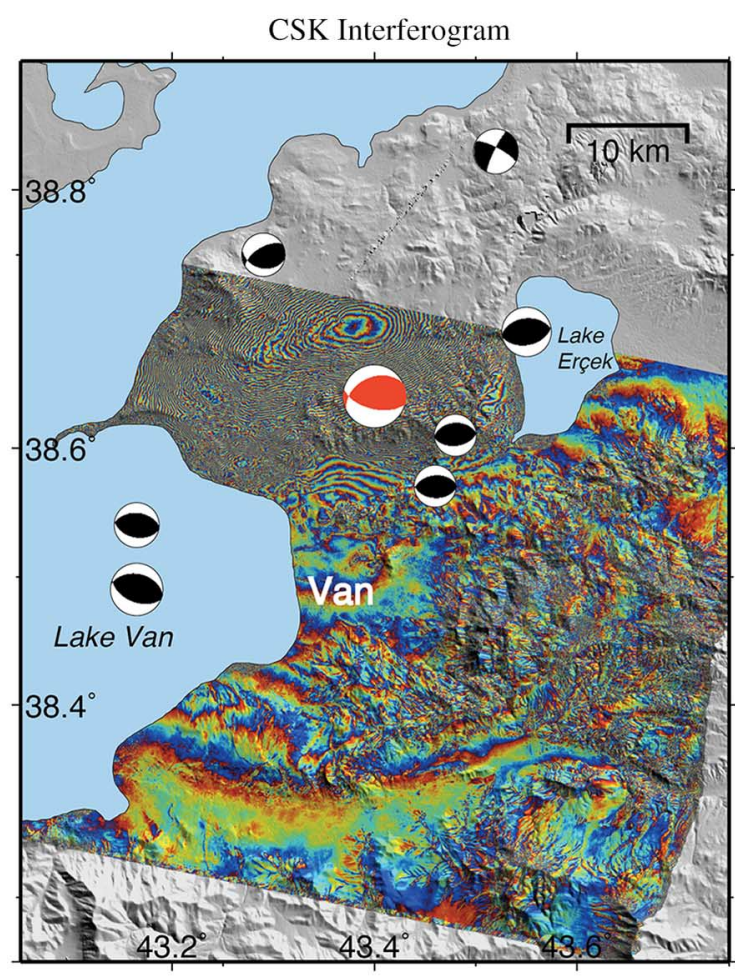

(a)
TSX Interferogram

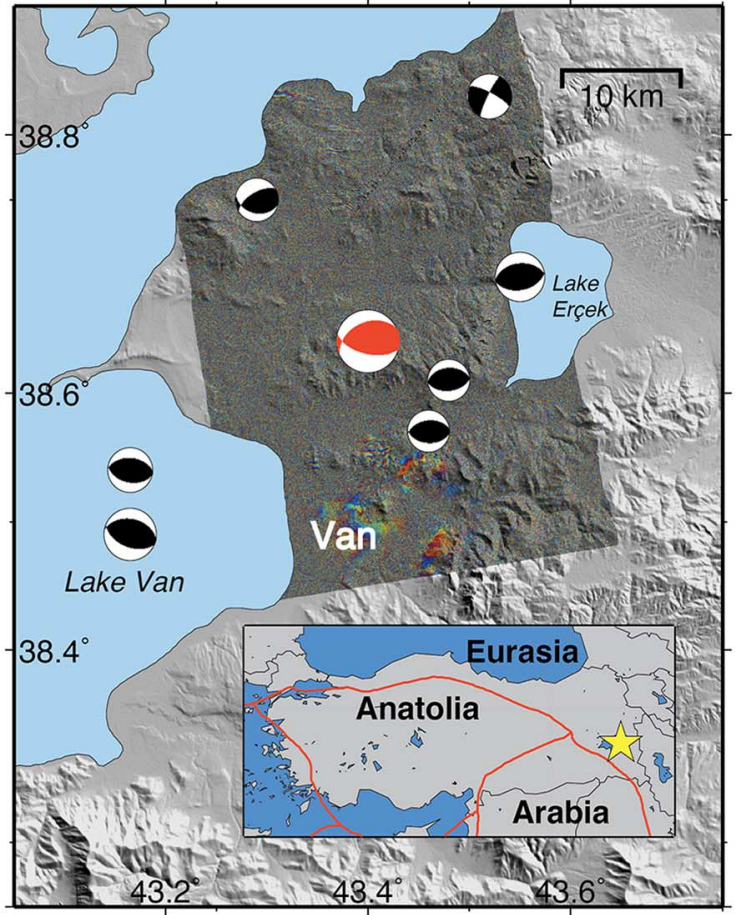

(b)

Fig. 1. Coseismic interferograms of the 23 October 2011 Van earthquake from (a) COSMO-SkyMed (CSK) descending-orbit images acquired on 10 October 2011 and 23 October 2011, with a perpendicular baseline of $192 \mathrm{~m}$ and (b) ascending TerraSAR-X (TSX) images acquired on 29 March 2009 and 31 October 2011, with a baseline of $36 \mathrm{~m}$. The global CMT solutions of the mainshock (in red) and aftershocks before 31 October 2011 (larger than $M_{w} 4.8$ ) are shown. The inset in (b) shows the location of the study area in eastern Turkey with the plate boundaries shown as red lines.

geological processes, rather than the X-band InSAR data, e.g., in [15]-[17].

Most SAR pixel offset applications use the conventional offset-measurement method, which is based on crosscorrelating regularly spaced subimages (windows) throughout the imaged area, although it does not take into consideration the variety of scattering characteristics on the ground. Here, we show how coseismic displacement mapping from SAR pixel offsets can be improved by focusing the offset measurements on strong reflecting targets, which allows retrieval of useful displacement information even when the corresponding interferogram appears to be completely decorrelated. The strategy has already been applied to improve SAR image coregistration for InSAR applications [18], [19] and initial applications to medium-resolution SAR images for coseismic displacement mapping have shown encouraging results [20], prompting us to develop this technique further and to evaluate its advantages more extensively. Using high-resolution SAR data from the 2011 Van earthquake in eastern Turkey, we demonstrate significant improvements from using our proposed method compared with the conventional offset-measurement method. We are also able to derive the first 3-D coseismic surface displacement field for the earthquake.

\section{INSAR DATA OF THE VAN EARTHQUAKE}

InSAR data played a key role in constraining the source model of the $M_{w} 7.1$ Van earthquake in eastern Turkey, which occurred on 23 October 2011 and caused over 600 casualties [1], [21]. The main data source in studies of the Van earthquake was a single descending CSK interferogram that provided details of the deformation in the epicentral area between Van and Erçek lakes, north of Van city [Fig. (1a)]. However, due to the CSKs short wavelength (one interferometric fringe represents $\sim 1.5 \mathrm{~cm}$ displacement in the LOS direction), the interferogram includes very dense fringes on the uplifting hanging wall, making phase unwrapping challenging. Despite this challenge, both Fielding et al. and Elliott et al. carefully unwrapped the interferogram and reported that the relative LOS displacement between the strongly uplifting hanging-wall and subsiding footwall exceeded $1 \mathrm{~m}$ and that the earthquake took place on a steep north-dipping thrust fault, located just north of Van city [1], [21]. However, while both groups found the fault dip to be $\sim 45^{\circ}$ to the north, they markedly differed on the two fault slip distributions, with Fielding et al. showing a significant amount of slip reaching to the surface [1], while Elliott et al. found that the slip was confined below $8 \mathrm{~km}$ depth [21].

Ascending TSX data of the earthquake also exist, but these data were not used in the earlier studies, because the coseismic TSX interferogram is almost completely decorrelated [Fig. 1(b)]. The only preseismic TSX image is from 29 March, 2009 , or from more than 2.5 years before the earthquake, a period of time that appears to be too long to retain coherence in the area. The only visible fringes in the TSX interferogram are found within Van city [Fig. 1(b)], making the data unusable for coseismic modeling. Loss of interferometric correlation 
is sometimes caused by large ground displacements [12], but in this case it is due to temporal decorrelation. Given the importance of having both ascending and descending data of coseismic deformation to reduce fault model parameter tradeoffs [2], we consider whether or not some usable displacement information can be extracted from this dataset.

\section{SAR OfFSET MEASUREMENTS ON PREDETECTED TARGETS}

The standard SAR image offset tracking method estimates offsets using cross-correlation between image windows (e.g., $64 \times 64$ pixels) that are uniformly distributed throughout the SAR image pair [3]. This strategy works well in areas where interferometric coherence is high, e.g., in arid desert regions. However, uniform distribution of cross-correlation windows may be far from optimal. There are two main drawbacks of such window distribution: 1) many windows could be in decorrelated areas, e.g., in lakes or agricultural fields, and some of these windows might show high correlation values because of the overestimation effect [22], complicating the selection of reliable measurements; and 2) strong reflectors (e.g., point-like targets) cause patch-like offset results. The latter is because the measured offset is a summation of all the pixel contributions within the cross-correlation windows, and each contribution is weighted by its reflective strength [23]. As a consequence, when a single strong reflector dominates the offset estimation of several neighboring and overlapping windows, similar offset estimations emerge and patch-like offset patterns appear (Fig. 2). Eliminating this effect is not straight forward, because offset measurements containing a strong reflector show high cross-correlation values and high signal-to-noise ratios (SNRs).

To improve the reliability and accuracy of SAR image offset estimations, we first identify strong reflectors (e.g., point-like targets) and then focus the offset estimations on them, instead of uniformly distributing the cross-correlation windows throughout the images. We cross-correlate a 2-D sinc-function template [Fig. 2(a), inset] with the SAR image to detect and locate the strong targets, as such targets cause 2-D sinc-function patterns in SAR amplitude images due to side-lobes of the transmitted SAR beam, e.g., in [18]. We then multiply the resulting cross-correlation map by the SAR amplitude image itself to suppress amplitudes of other pixels than the detected targets [Fig. 2(b)]. Using a similar strategy, Hu et al. [20] simply selected bright pixels using a certain threshold amplitude value, which is useful for avoiding decorrelated areas (e.g., water bodies), but often yields patch-like offset estimations, because strong reflectors commonly cluster in space and strong side lobes may be selected. To avoid this problem, we search for local maxima in the enhanced amplitude map and discard pixels with image-sinc cross-correlation values lower than certain thresholds (e.g., 0.2). We ensure that some offset measurement locations have been selected throughout the whole image by applying this target detection strategy on overlapping blocks with adaptive threshold values, determined from the local mean $\mu$, and the standard deviation (STD) $\sigma$ of the enhanced amplitude values, i.e., strong reflectors are selected if the amplitude exceeds $\mu+2 \sigma$. When several pre or postseismic images are

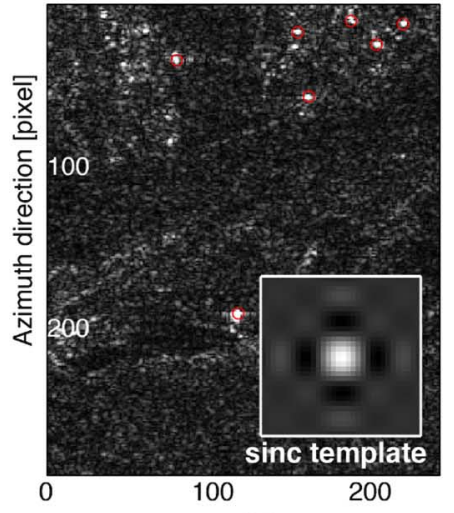

(a)

(c)

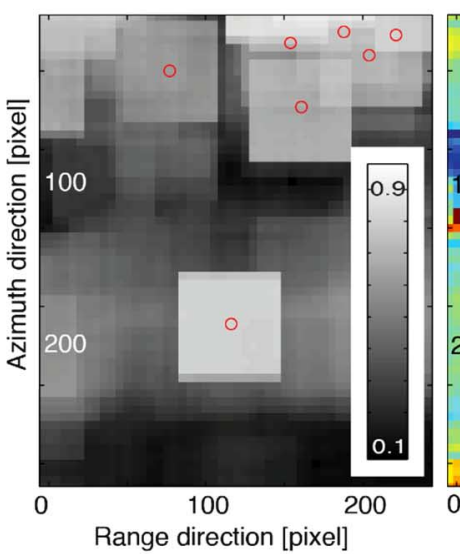

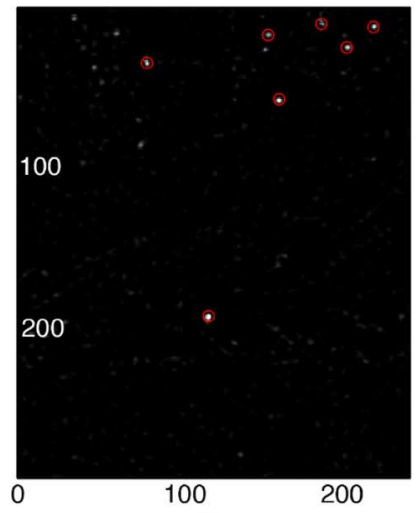

(b)

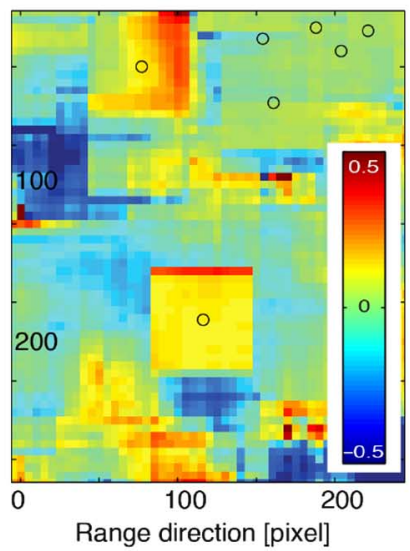

(d)
Fig. 2. Example of point-target detection and patch-like results from standard image offset processing. (a) and (b) The original and enhanced amplitude maps, respectively, for a small area near Van city. Point-like targets, detected using a "sinc" template (inset in a), are shown as circles. (d) Azimuth image offsets determined from the standard method and estimated using (c) Cross-correlation of image windows (here $64 \times 64$ pixels, with a step of 5 pixels), producing patch-like offset results. (a) SAR amplitude image. (b) Enhanced amplitude image. (c) Cross-correlation map. (d) Azimuth offsets.

available, we can first co-register the images and then locate the point-like targets from an averaged amplitude map, which yields better results than using only a single SAR image [19]. Some of the detected targets are probably not point targets, but bright distributed targets or reflectors showing strong reflectivity, e.g., dihedral reflectors. For the sake of simplicity, in the following discussion we will still use the term point-target offsets in referring to the offsets calculated on the detected targets.

We applied this target detection strategy to the CSK and TSX datasets of the Van earthquake. We coregistered three postseismic TSX images (acquired on 31 October 2011, and on 11 and 25 November 2011), and used the averaged amplitude map to detect reflectors. Note that averaging multiple images does not help when displacements larger than a pixel occur during the time span of the co-registered dataset, which is not the case here. As expected, the highest density of detected strong reflectors is found within Van city and in the neighboring villages (Fig. S1). We then estimated both the azimuth and range offsets between the pre and postearthquake images 


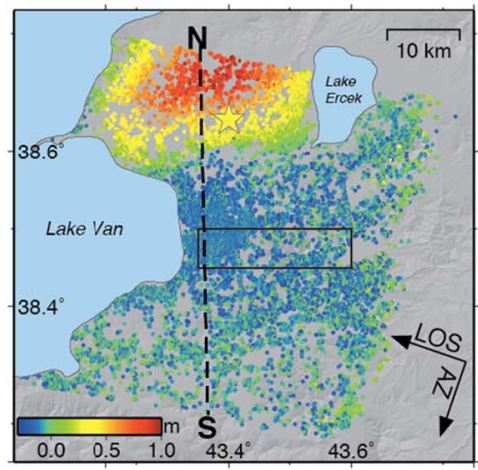

(a)

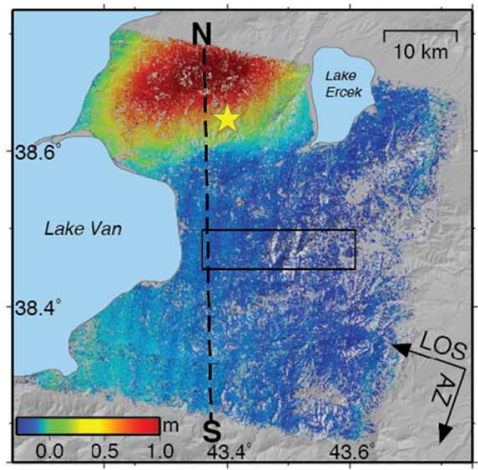

(e)

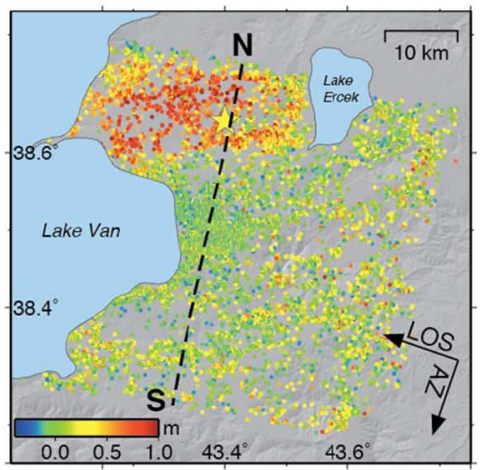

(b)

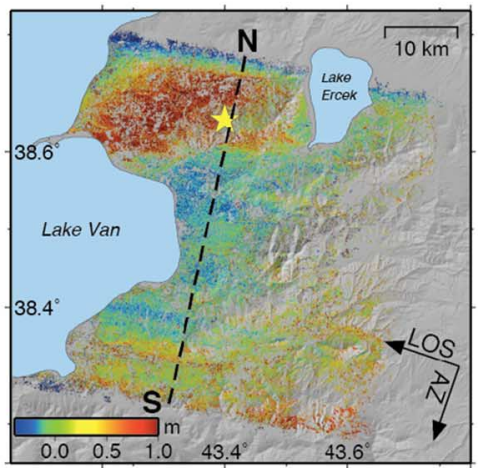

(f)

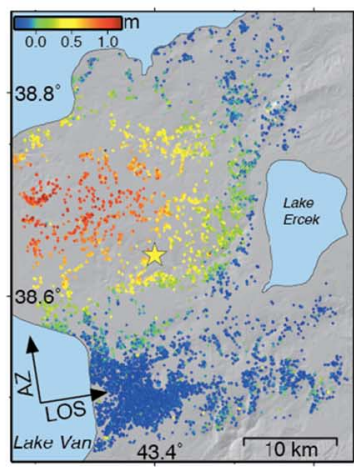

(c)

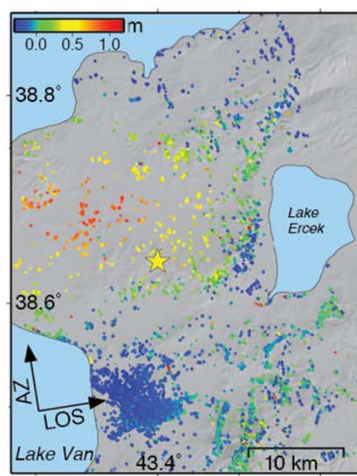

(g)

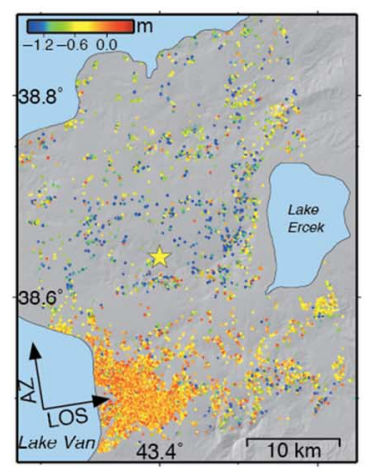

(d)

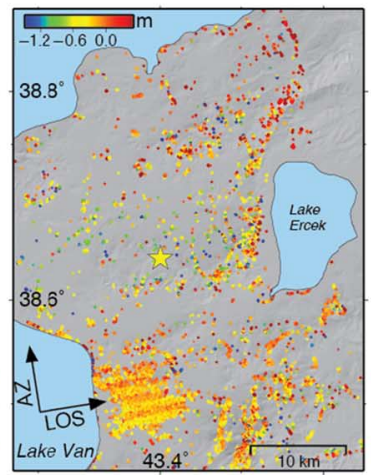

(h)

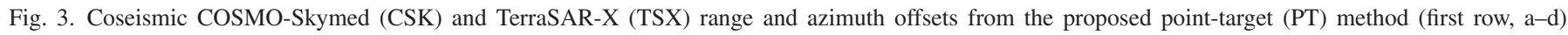

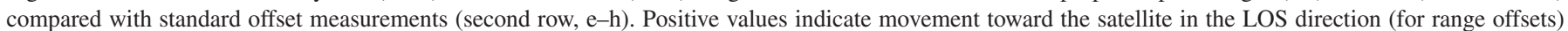

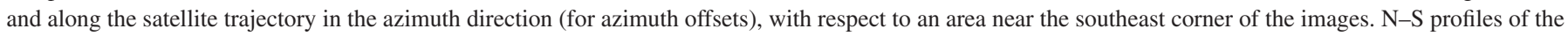

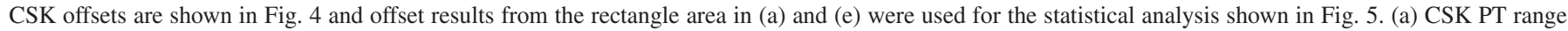

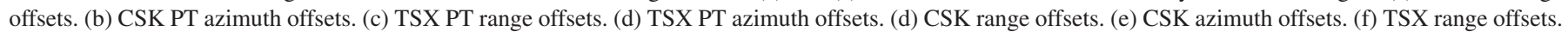
(g) TSX azimuth offsets.

by cross-correlating $64 \times 64$ pixel windows centered on the detected targets. We also calculated orbital offsets for each window from the reported orbital information and a digital elevation model (DEM) (Figs. S2 and S3), as the difference between the amplitude- and orbit-derived offsets reveals the coseismic displacement. The CSK range and azimuth offset results show a meter-level uplift and southward displacement, respectively, of the hanging wall north of Van city [Fig. 3(a) and (b)]. The uplift is also clearly revealed by the TSX results using the proposed offset method [Fig. 3(c) and (d)], even though the corresponding TSX interferogram is completely decorrelated [Fig. 1(b)].

\section{COMPARISON With STANDARD SAR OFFSET MEASUREMENTS}

To compare our offset estimation results with standard image-offset measurements, we processed the same datasets using the offset-tracking package of the GAMMA software [24]. We uniformly distributed overlapping $64 \times 64$ pixel windows (10-pixel step size) across the images and estimated the azimuth and range offsets from the cross-correlation, which resulted in $100 \times$ more cross-correlation calculations than in the proposed approach described above. We then geocoded the offset map and removed offset estimations with low SNR $(<10)$
[Fig. 3(e)-(h)] before comparing them with the point-target offset results [Fig. 3(a)-(d)].

The estimated offsets using the standard method include signals from both ground displacements and variations in the orbital geometry. For mid-resolution SAR images, these orbitinduced offsets can usually be mostly removed by fitting a surface to the offset measurements in nondeforming areas, when the spatial baseline is small. In high-resolution data, however, the range offsets are much more sensitive to the topography [Figs. S2(b), (d), S3(b), and (d)] [25]. The strong correlation between the range offsets and topography can clearly be seen when inspecting the offset results along a north-south profile from the hanging wall and through Van city located on the footwall [Fig. 4(a), blue line]. Therefore, removing topographic signals from X-band SAR offset measurements is essential before the results can be used in geoscientific applications.

We removed the orbit-induced offsets using a DEM and accurate orbital information, i.e., by first calculating the expected orbital offsets between the pre and postseismic images and then by removing them from the standard offset results [26]. This correction significantly improves the standard offsets, making them comparable to the point-target offset results in the highly correlated CSK case [Fig. 4(a)]. We then compared the CSK range offsets with the unwrapped InSAR data using the southeastern image corner as reference, which provide an 


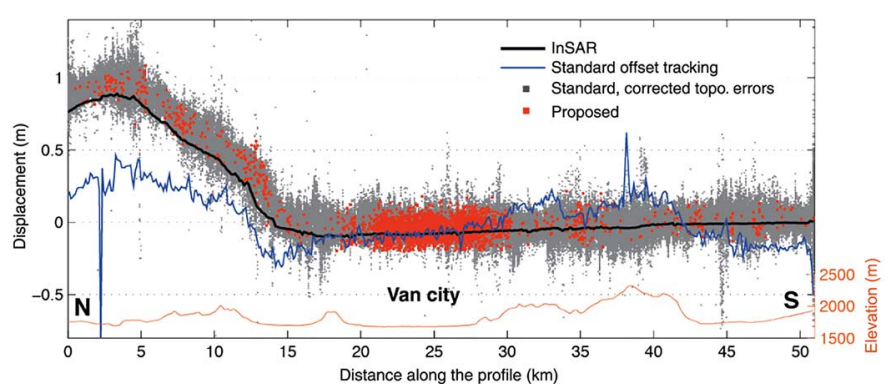

(a)

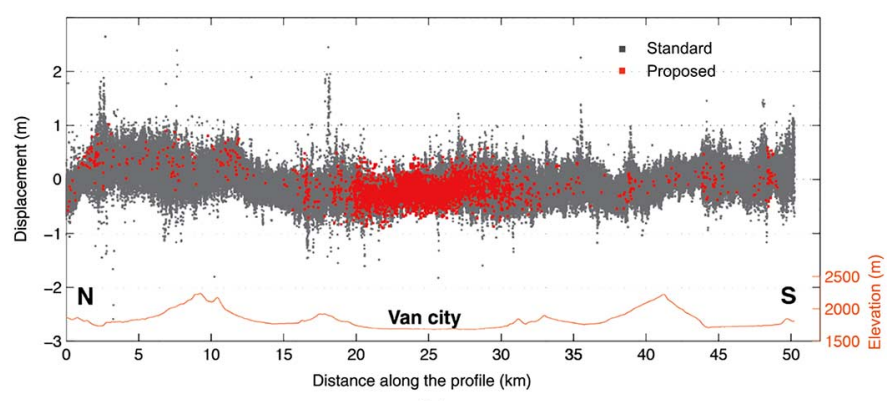

(b)

Fig. 4. Coseismic COSMO-Skymed (a) range and (b) azimuth offsets from the standard and the proposed methods along an N-S profile [Fig. 3(a)-(b) and (e)-(f)]. In (a), the range offsets are compared with InSAR.

independent and normally more accurate measurement along the same LOS direction (Fig. S4). However, both offset profiles show stronger displacement maxima than InSAR, which is probably due to InSAR phase unwrapping errors in the area with the highest displacement gradients, leading to an underestimation of the deformation from InSAR.

To validate the results quantitatively, we analyzed window cross-correlation values and calculated STD of the differences between the InSAR data and the point-target and standard offset results within an area where no InSAR unwrapping errors are expected [the rectangles in Fig. 3(a) and (e)]. First, the proportion of high cross-correlation values is clearly larger for the point-target offsets than for the offsets from the standard method [Fig. 5(a)], indicating that the point-target offset determinations are of better quality. Then, after removing a few obvious outliers from both results (offsets beyond $\pm 1 \mathrm{~m}$ ), we find that the STD between the point-target offsets and InSAR results is $6.93 \mathrm{~cm}$, which is only about half of the corresponding STD value for the standard offsets $(12.6 \mathrm{~cm})$. Using a higher SNR offset-threshold leads to better, but fewer standard offset measurements. Ranging the SNR thresholds from 7 to 30 yields the best results with a threshold of SNR $=18$, approaching that of the point-target results [Fig. 5(b)]. However, with such a high SNR threshold, only offsets surrounding the strongest reflectors remain, resulting in patch-like offset measurements and greatly reduced areal coverage (Fig. S5). Note that the STD does not monotonically decrease with increasing SNR threshold [Fig. 5(b)], because the influence of remaining offsetoutliers increases when the number of offset estimations is significantly reduced (with a higher SNR threshold). In contrast, much fewer outliers appear in the point-target offset map, as we only calculate the offsets on predetected strong reflectors.
In comparison, it is noteworthy that the STD of the point-target offsets does not depend strongly on the sinc-correlation threshold [Fig. 5(c)], implying that the point-target offsets contain less outliers and are therefore more reliable for mapping the surface displacements.

Another problem with the offset results from the standard method is that they, particularly the azimuth offsets, exhibit band-like artifacts [Figs. 3(f), (h), and 4(b)]. This phenomenon is caused by squint-angle variations between the pre and postseismic SAR images [27]. These variations result in reduced Doppler spectral overlap between SAR images and are difficult to model or remove. Consequently, the CSK azimuth offsets are only useful where the displacements are large [1]. However, squint-angle variations hardly affect the signal reflected back from ideal point-targets [28] and band-like artifacts are therefore significantly reduced in the point-target offset results [Fig. 3(b) and (d), inside Van city].

\section{3-D COSEISMiC Displacements}

The complete 3-D coseismic displacement field can be estimated in areas where the CSK and TSX images overlap, i.e., in the area between Van and Erçek lakes and in Van city. The system of linear equations linking the azimuth- and range-offset measurements ( $d_{a z}$ and $\left.d_{l o s}\right)$ to 3-D surface displacements $U$, is [6], [29]

$$
\left[\begin{array}{ccc}
\sin \theta^{c} \sin \phi^{c} & -\sin \theta^{c} \cos \phi^{c} \cos \theta^{c} \\
\sin \theta^{t} \sin \phi^{t} & -\sin \theta^{t} \cos \phi^{t} & \cos \theta^{t} \\
\cos \phi^{c} & \sin \phi^{c} & 0 \\
\cos \phi^{t} & \sin \phi^{t} & 0
\end{array}\right]\left[\begin{array}{c}
U_{e} \\
U_{n} \\
U_{u}
\end{array}\right]=\left[\begin{array}{c}
d_{\text {los }}^{c} \\
d_{\text {los }}^{t} \\
d_{a z}^{c} \\
d_{a z}^{t}
\end{array}\right]
$$

where $\theta$ is the radar incidence angle, $\phi$ is the satellite heading angle, and superscripts $c$ and $t$ correspond to the CSK and TSX satellites, respectively. Using this system of equations, we computed the 3-D displacements where at least three measurements exist within a $2 \mathrm{~km} \times 2 \mathrm{~km}$ window that was moved across the imaged area (Fig. 6). The ascending TSX data span a much longer time period (29 March 2009-31 October 2011) than the descending CSK data (13-23 October 2011) and could therefore potentially include more pre and postseismic deformation. However, there were no large earthquakes (larger than $M_{w} 4.8$ ) in the area from 2009 and until the Van earthquake occurred on 23 October, 2011. The largest aftershock $\left(M_{w} 5.9\right)$, on the other hand, occurred $10 \mathrm{~h}$ after the mainshock and after the second CSK acquisition. Deformation due to this event, and other smaller aftershocks (Fig. 1) in the following 8 days, is therefore only included in the TSX data. According to Elliott et al. [21], the largest aftershock caused only about $7 \mathrm{~cm}$ uplift, far smaller than the deformation caused by the mainshock (more than $1 \mathrm{~m}$ uplift). We therefore ignore the postseismic displacements in the 3-D displacement derivation.

The derived 3-D displacement map covers the transition from the hanging wall in the north to the footwall in the south, showing a clear belt of high displacement gradients between the two lakes. On the hanging wall, the displacement pattern is dominated by a strong uplift exceeding $1 \mathrm{~m}$, while about $10 \mathrm{~cm}$ subsidence is found in Van city. The horizontal displacements 


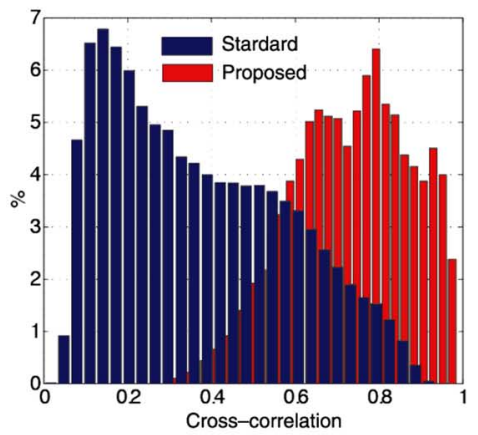

(a)

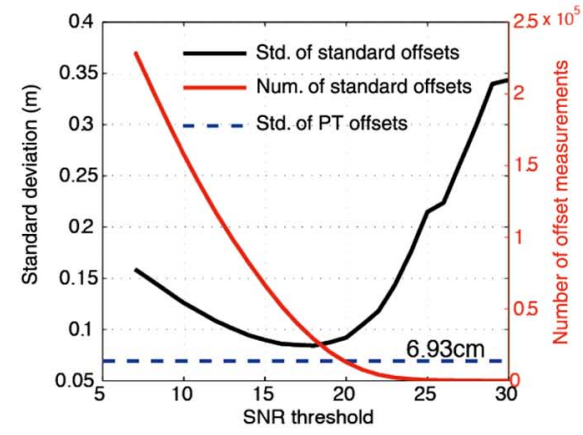

(b)

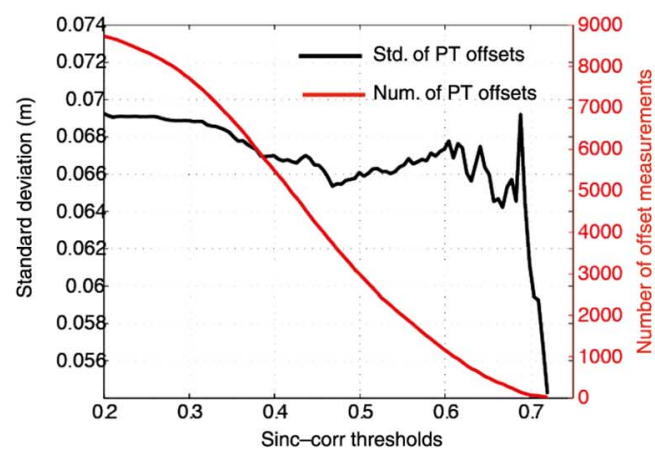

(c)

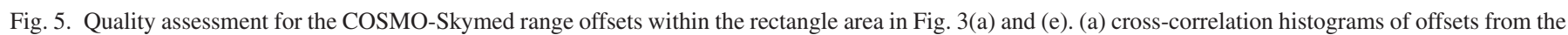

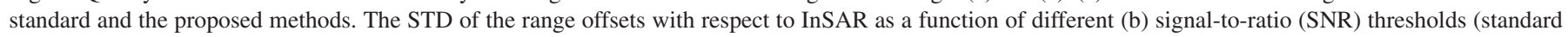
method) and (c) sinc-correlation thresholds (proposed method).

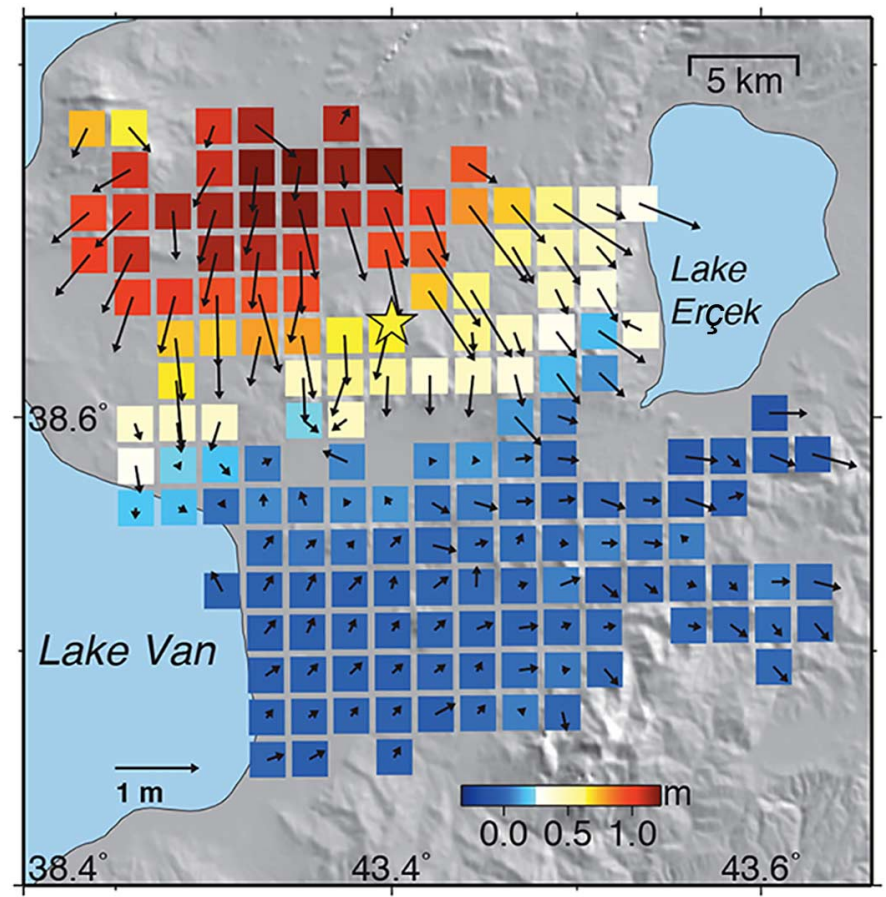

Fig. 6. 3-D coseismic displacement map for the Van earthquake derived from the offset measurements with arrows showing the horizontal and colors the vertical displacements. The colored patches are $2 \mathrm{~km} \times 2 \mathrm{~km}$ in size, from which the 3-D displacements were calculated.

exhibit about $1 \mathrm{~m}$ of north-south convergence with the strongest displacements occurring on the hanging-wall block. Overall, the derived 3-D displacements show the expected deformation pattern from a steeply north-dipping and a blind thrust fault [21], but they do not indicate that the fault slip reached the surface as implied by the model of [1].

\section{DiscuSSION AND CONCLUSION}

Deriving the complete 3-D coseismic displacement field near the epicenter of an earthquake is important to constrain fault slip models. Researchers have estimated 3-D displacements using InSAR data alone, e.g., in [29], InSAR and azimuth offsets, e.g., in [7], InSAR and optical image offsets, e.g., in [30], as well as InSAR and multiple aperture interferometry (MAI), e.g., in [31]. In such cases, coseismic interferograms have to exhibit high coherence values and be correctly unwrapped before the 3-D displacement derivation can be applied. However, neither InSAR nor MAI can provide useful information from the TSX images of the Van earthquake, as the decorrelation due to ground changes is too severe. While other studies using radar offsets alone [8] (or radar and optical offsets, e.g., in [32]) to map 3-D coseismic displacements have been carried out, they focused on cases where large surface ruptures took place, causing multimeter surface displacement discontinuities. The Van earthquake case is different as the deformation signal was smooth with no sharp fault rupture because the causative fault did not reach the surface. The presented results demonstrate that offsets from predetected targets, rather than the InSAR phase, can be used to measure ground displacement in cases when X-band interferograms are severely decorrelated or when phase unwrapping is challenging.

The derived 3-D coseismic displacement map of the Van earthquake reveals strong uplift and an interesting pattern of divergent horizontal displacements on the hanging wall (Fig. 6). This type of displacement pattern has also been observed in several other reverse faulting earthquakes where a dense GPS network is available, e.g., in the 1999 Chi-Chi and the 2003 Chengkung earthquakes in Taiwan [33], [34]. Hampel et al. [35] used a 3-D numerical model to study the expected deformation near the fault tips of thrust and normal faults. Their results show divergent horizontal displacements on the hanging wall away from the area of maximum uplift. These displacement characteristics are consistent with the offset-derived displacement vectors of the Van earthquake (Fig. 6). 3-D displacement maps from SAR images provide measurements at a much higher density than does GPS, allowing identification of unprecedented deformation details near the causative fault tips.

The main drawback of the proposed method is that the measurement improvement depends on the density of strong reflectors in the area of interest, which are mainly man-made point-like targets in the Van case. In rural areas with fewer or even no man-made structures, we might only be able to detect a small number of good reflectors, possibly leaving large areas without any measurements. Nevertheless, if there are some 
natural targets with strong reflectivity, we can still use these targets as supplementary measurements to calibrate or to improve results based on the standard method.

For the time from the loss of the Envisat and ALOS satellites in early 2011 until the launch of Sentinel-1A and ALOS-2 satellites in 2014, images from the TSX and CSK satellites are the main source for SAR deformation measurements. However, $\mathrm{X}$-band interferograms often completely lose coherence after a year or more in moderately vegetated areas. For such cases, the method presented here offers a new strategy for obtaining coseismic displacement measurements from SAR images, even if the corresponding interferograms appear to be completely decorrelated. Time-series analysis of SAR offset measurements has been proposed and successfully applied to the analysis of volcanic deformation and landslide motion, e.g., in [36] and [17]. For pixel-offset series on detected point-like targets, such time-series deformation analysis should work even better, as indicated by recent results from image coregistration [19]. In this way, the amplitude information in high-resolution SAR images allows us to map deformation due to a variety of geophysical phenomena with accuracy of a few centimeters (validated from InSAR) on the detected good reflectors.

\section{ACKNOWLEDGMENT}

The point-target offset results and the 3-D coseismic displacements shown in Figs. 3 and 6, respectively, can be found in the Supplementary Information. MATLAB functions to detect point-like targets in SAR images and to correct for topographic effects are available upon request. The CSK SAR data were provided by the Italian Space Agency and the TSX data were obtained from the GEO SuperSites project. The research reported in this publication was supported by King Abdullah University of Science and Technology (KAUST). The authors would like to thank Guangcai Feng (KAUST), for calculating the offsets with the GAMMA software and Ahmet M. Akoglu (KAUST), for useful discussions.

\section{REFERENCES}

[1] E. J. Fielding, P. R. Lundgren, T. Taymaz, S. Yolsal-evikbilen, and S. E. Owen, "Fault slip source models for the 2011 M7.1 Van earthquake in Turkey from SAR interferometry, pixel offset tracking, GPS and seismic waveform analysis," Seismol. Res. Lett., vol. 84, no. 4, pp. 579-593, 2013.

[2] H. Sudhaus and S. Jónsson, "Improved source modeling through combined use of InSAR and GPS under consideration of correlated data errors: Application to the June 2000 Kleifarvatn earthquake Iceland," Geophys. J. Int., vol. 176, pp. 389-404, 2009.

[3] R. Michel, J.-P. Avouac, and J. Taboury, "Measuring ground displacements from SAR amplitude images: Application to the Landers earthquake,” Geophys. Res. Lett., vol. 26, no. 7, pp. 875-878, 1999.

[4] R. Bamler and M. Eineder, "Accuracy of differential shift estimation by correlation and split-bandwidth interferometry for wideband and delta-k SAR systems," IEEE Geosci. Remote Sens. Lett., vol. 2, no. 2, pp. 151155, Apr. 2005.

[5] F. De Zan, "Accuracy of incoherent speckle tracking for circular Gaussian signals," IEEE Geosci. Remote Sens. Lett., vol. 11, no. 1, pp. 264-267, Jan. 2014 .

[6] Y. Fialko, M. Simons, and D. Agnew, "The complete (3-D) surface displacement field in the epicentral area of the $1999 \mathrm{Mw} 7.1$ Hector Mine earthquake, California, from space geodetic observations," Geophys. Res. Lett., vol. 28, no. 16, pp. 3063-3066, 2001.
[7] Y. Fialko, D. Sandwell, M. Simons, and P. Rosen, "Three-dimensional deformation caused by the Bam, Iran, earthquake and the origin of shallow slip deficit," Nature, vol. 435, pp. 295-299, 2005.

[8] E. Pathier et al., "Displacement field and slip distribution of the 2005 Kashmir earthquake from SAR imagery," Geophys. Res. Lett., vol. 33, p. L20310, 2006, doi: 10.1029/2006GL027193.

[9] M. De Michele et al., "Three dimensional surface displacement of the 2008 May 12 Sichuan earthquake (China) derived from synthetic aperture radar: Evidence for rupture on a blind thrust," Geophys. J. Int., vol. 183, no. 3, pp. 1097-1103, 2010.

[10] S. Jónsson, H. Zebker, and F. Amelung, "On trapdoor faulting at Sierra Negra volcano, Galápagos,” J. Volcanol. Geotherm. Res., vol. 144, no. 1-4, pp. 59-71, 2005.

[11] J. L. Elliott, J. T. Freymueller, and B. Rabus, "Coseismic deformation of the 2002 Denali fault earthquake: Contributions from synthetic aperture radar range offsets," J. Geophys. Res., vol. 112, p. B06421, 2007, doi: 10.1029/2006JB004428.

[12] S.-H. Yun, H. Zebker, P. Segall, A. Hooper, and M. Poland, "Interferogram formation in the presence of complex and large deformation," Geophys. Res. Lett., vol. 34, p. L12305, 2007, doi: 10.1029/2007GL029745.

[13] Y. Yan et al., "Coseismic slip distribution of the 2005 Kashmir earthquake from SAR amplitude image correlation and differential interferometry," Geophys. J. Int., vol. 193, no. 1, pp. 29-46, 2013, doi: $10.1093 / \mathrm{gji} / \mathrm{ggs} 102$.

[14] M. Eineder, C. Minet, P. Steigenberger, X. Cong, and T. Fritz, "Imaging geodesy-toward centimeter-level ranging accuracy with TerraSAR-X," IEEE Trans. Geosci. Remote Sens., vol. 49, no. 2, pp. 661-671, Jan. 2011.

[15] R. Fallourd et al., "Monitoring temperate glacier displacement by multitemporal TerraSAR-X images and continuous GPS measurements,' IEEE J. Sel. Topics Appl. Earth Observ. Remote Sens., vol. 4, no. 2, pp. 372386, May 2011.

[16] N. Yague-Martinez, M. Eineder, X. Cong, and C. Minet, "Ground displacement measurement by TerraSAR-X image correlation: The 2011 Tohoku-Oki earthquake," IEEE Geosci. Remote Sens. Lett., vol. 9, no. 4, pp. 539-543, Jun. 2012.

[17] D. Raucoules, M. De Michele, J.-P. Malet, and P. Ulrich, "Time-variable 3D ground displacements from high-resolution synthetic aperture radar (SAR): Application to La Valette landslide (South French Alps),' Remote Sens. Environ., vol. 139, pp. 198-204, 2013.

[18] F. Serafino, "SAR image coregistration based on isolated point scatterers," IEEE Geosci. Remote Sens. Lett., vol. 3, no. 3, pp. 354-358, Jul. 2006.

[19] T. Wang, S. Jónsson, and R. F. Hanssen, "Improved SAR image coregistration using pixel-offset series," IEEE Geosci. Remote Sens. Lett., vol. 11, no. 9, pp. 1465-1469, Mar. 2014.

[20] X. Hu, T. Wang, and M. Liao, "Measuring coseismic displacements with point-like targets offset tracking," IEEE Geosci. Remote Sens. Lett., vol. 11, no. 1, pp. 283-287, Jan. 2014.

[21] J. R. Elliott, A. C. Copley, R. Holley, K. Scharer, and B. Parsons, "The $2011 \mathrm{Mw} 7.1$ Van (eastern Turkey) earthquake," J. Geophys. Res., vol. 118, pp. 1619-1637, 2013.

[22] H. A. Zebker and K. Chen, "Accurate estimation of correlation in InSAR observations," IEEE Geosci. Remote Sens. Lett., vol. 2, no. 2, pp. 124-127, Apr. 2005.

[23] R. Bamler and P. Hartl, "Synthetic aperture radar interferometry," Inverse Probl., vol. 14, no. 4, p. R1, 1998, doi: 10.1088/0266-5611/14/4/001.

[24] C. Werner et al., "Gamma SAR and interferometric processing software," in Proc. ERS-ENVISAT Symp., Gothenburg, Sweden, 2000.

[25] D. O. Nitti, R. F. Hanssen, A. Refice, F. Bovenga, and R. Nutricato, "Impact of DEM-assisted coregistration on high-resolution SAR interferometry," IEEE Trans. Geosci. Remote Sens., vol. 49, no. 3, pp. 1127-1143, Feb. 2011.

[26] E. Sansosti, P. Berardino, M. Manunta, F. Serafino, and G. Fornaro, "Geometrical SAR image registration," IEEE Trans. Geosci. Remote Sens., vol. 44, no. 10, pp. 2861-2870, Sep. 2006.

[27] M. Boncori, J. Peter, and P. Giuseppe, "Performance of COSMO-SkyMed split bandwidth interferometry for the measurement of co-seismic displacement," in Proc. Living Planet Symp., Edinburgh, U.K., 2013.

[28] D. Perissin, C. Prati, M. E. Engdahl, and Y. L. Desnos, "Validating the SAR wavenumber shift principle with the ERS-Envisat PS coherent combination," IEEE Trans. Geosci. Remote Sens., vol. 44, no. 9, pp. 2343-2351, Aug. 2006.

[29] T. J. Wright, B. E. Parsons, and Z. Lu, "Toward mapping surface deformation in three dimensions using InSAR," Geophys. Res. Lett., vol. 31, p. L01607, 2004, doi: 10.1029/2003GL018827. 
[30] I. Barisin, S. Leprince, B. Parsons, and T. Wright, "Surface displacements in the September 2005 Afar rifting event from satellite image matching: Asymmetric uplift and faulting," Geophys. Res. Lett., vol. 36, p. L07301, 2009, doi: 10.1029/2008GL036431.

[31] S. Jónsson, "Tensile rock mass strength estimated using InSAR," Geophys. Res. Lett., vol. 39, p. L21305, 2012, doi: 10.1029/2012GL 053309.

[32] J. Harrington et al., "Coseismic surface displacements from optical and SAR image offset tracking, fault modeling and geomorphological analysis of the Sept. 24th, 2013 M7.7 Balochistan earthquake," presented at the General Assembly, European Geosciences Union, Vienna, Austria, Apr. 27-May 02, 2014

[33] J.-C. Lee et al., "Quantitative analysis of surface coseismic faulting and postseismic creep accompanying the 2003, $\mathrm{Mw}=6.5$, Chengkung earthquake in eastern Taiwan," J. Geophys. Res.: Solid Earth, vol. 111, no. B2, 2006, doi: 10.1029/2005JB003612.

[34] Y. J. Hsu, S. B. Yu, and H. Y. Chen, "Coseismic and postseismic deformation associated with the 2003 Chengkung, Taiwan, earthquake," Geophys. J. Int., vol. 176, no. 2, pp. 420-430, 2009.

[35] A. Hampel, T. Li, and G. Maniatis, "Contrasting strike-slip motions on thrust and normal faults: Implications for space-geodetic monitoring of surface deformation," Geology, vol. 41, no. 3, pp. 299-302, 2013.

[36] F. Casu, A. Manconi, A. Pepe, and R. Lanari, "Deformation timeseries generation in areas characterized by large displacement dynamics: The SAR amplitude pixel-offset SBAS technique," IEEE Trans. Geosci. Remote Sens., vol. 49, no. 7, pp. 2752-2763, Jun. 2011.

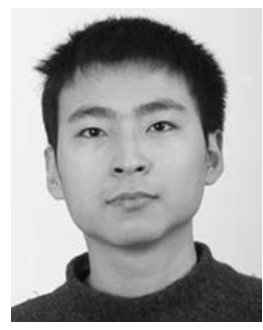

Teng Wang was born in Xinxiang, China, in 1980 $\mathrm{He}$ received the B.Eng. and M.Eng. degrees in photogrammetry and remote sensing from Wuhan University, Wuhan, China, in 2003 and 2006, respectively, and the Ph.D. degrees in information technology from Wuhan University and the Politecnico di Milano, Milan, Italy, in 2010.

Since October 2010, he has been working as a Postdoctoral Researcher with King Abdullah University of Science and Technology (KAUST), Thuwal, Saudi Arabia. His research interests include InSAR and offset tracking techniques, and their applications in geophysical studies.

Dr. Wang is a Member of the American Geophysical Union. He received the IEEE JSTARS Best Paper Award in 2012.

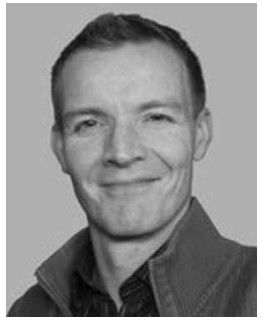

Sigurjón Jónsson was born in Húsavík, North Iceland, in 1971. He received the B.S. and M.S. degrees in geophysics from the University of Iceland, Reykjavík, Iceland, in 1994 and 1996, respectively, and the M.S. degree in electrical engineering and the $\mathrm{Ph} . \mathrm{D}$. degree in geophysics from Stanford University, Stanford, CA, USA, in 2002. He was awarded the Reginald A. Daly Postdoctoral Fellowship at the Department of Earth and Planetary Sciences, Harvard University, Cambridge, MA, USA, in 2002.

From 2004 to 2009 , he was a Senior Researcher and Lecturer with the Institute of Geophysics, Swiss Federal Institute of Technology (ETH) Zürich, Zürich, Switzerland, and currently holds an Associate Professorship in Geophysics with King Abdullah University of Science and Technology (KAUST), Thuwal, Saudi Arabia.

Dr. Jónsson is a Member of the American Geophysical Union and the European Geosciences Union. 